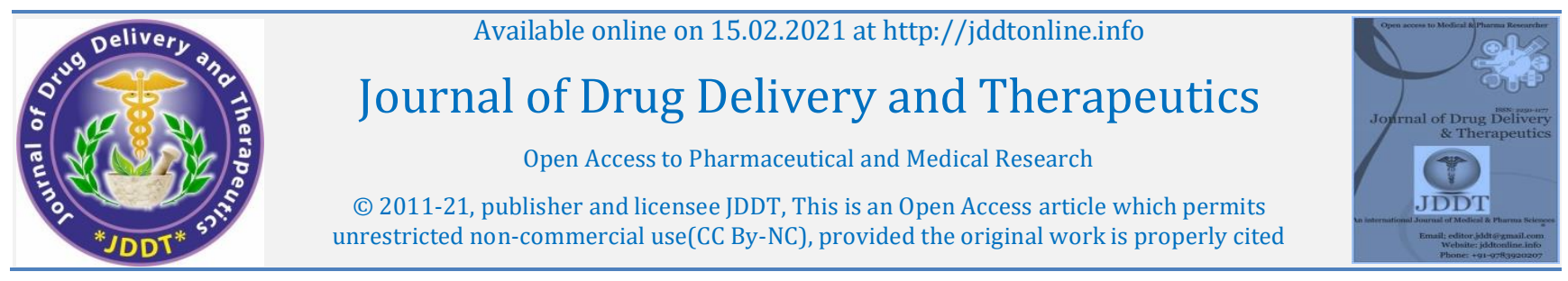

Open Access Full Text Article

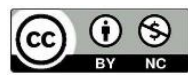

Research Article

\title{
An Evaluation of Medication Adherence to Antidiabetic Drugs: A Prospective Study
}

\author{
*Nimmy N John, V J Arjun, Darath David and H. Doddayya \\ NET Pharmacy College, Raichur-584103, Karnataka, India
}

\begin{abstract}
Article Info:
Abstract

Article History:

Received 08 Dec 2020;

Review Completed 19 Jan 2021

Accepted 27 Jan 2021;

Available online 15 Feb 2021

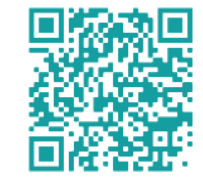

Adherence to prescribed medication is an imperative issue which can be directly linked with the management of diabetes mellitus. WHO identifies poor adherence is the most significant cause of uncontrolled blood sugar level. A prospective observational study was conducted in in-patients admitted to general medicine department diagnosed with diabetes mellitus from September 2019 to February 2020. Data was collected from case sheets of patients for age, gender, and socio- economic data. Informed consent was obtained from patients or bystanders before study. Direct interview was conducted with

Cite this article as: patients using standardized questionnaire known as MMAS-8. Patient's adherence to medications was assessed using scores obtained from the adherence scale. Among 100 in-patients $62(62 \%)$ patients were male and 38 (38\%) patients were female. Most of the patients had low adherence among both genders. Patients under age group of 41-60 years were more (58\%) in which $46 \%$ had low adherence. It was observed that, married patients were more with low adherence (58\%) followed by unmarried. When taking account of education of the patient, graduates and above were only 22 patients $(22 \%)$ but most of them (16\%) had moderate/high adherence. Education of the patients had a significant positive correlation (i.e., $\mathrm{p}$ value $=0.0000002$ ) between low adherence and high adherence of patients. This study showed that the adherence to anti-diabetic medications in diabetic patients were very less. It needs to be continuously evaluated in order to reduce complications and improve quality of life of patients.

Mrs. Nimmy N John, Assistant Prof essor, Department o Pharmacy Practice, NET Pharmacy College, Raichur-
\end{abstract}

Keywords: Medication adherence, MMAS-8, Uncontrolled diabetes

\section{INTRODUCTION:}

Diabetes mellitus is a metabolic vascular syndrome of multiple etiologies characterized by chronic hyperglycaemia with disturbance of carbohydrate, fat and protein metabolism resulting defects in insulin secretion, insulin action or both. This disorder is frequently associated with long term damage, which can lead to failure of organs like eyes, kidney, nerves, heart and blood vessels. According to International Diabetes Federation, $6.6 \%$ of the worldwide adult population had diabetes and it is estimated that by 2030, approximately $7.8 \%$ of the world wide adult population will have diabetes. In India the prevalence of diabetes has been increasing explosively over the last few years. In 2000 WHO reported that 32 million people was diagnosed with diabetes in India. India is poised to become the diabetic capital of the world with a patient population of 40.9 million in 2019, which is projected to increase to 69.9 million by 2025 . The prevalence was 2.1 per cent in urban population and 1.5 per cent in the rural population while in those above 40 year of age, the prevalence was 5 per cent in urban and 2.8 per cent in rural areas. 1,2

Adherence to prescribed medication is an imperative issue which can be directly linked with the management of chronic diseases like diabetes; failure to adherence can affect the effectiveness of medication as well as the efficiency of health care system. The maximal beneficial effect of an appropriate treatment plan can be achieved only if patients strictly adhere to the recommendations. ${ }^{3}$

Poor adherence to antidiabetic medications is an obstacle in the management of diabetes mellitus resulting in high rate of hospitalization and death. The world health organization (WHO) identifies poor adherence as the most significant reason for uncontrolled Blood glucose level, due to the indefinite treatment duration, medication adherence remains a significant challenge among the patient. Poor adherence is responsible for unnecessary numerous prescriptions of drugs, substantial worsening of diseases, increases in avoidable hospital admission rates and longer hospital case which all leading to a significant medical burden such as reduced optimal clinical benefit.4

One major factor that influences adherence is the patient's ability to read and understand medication instructions. Patients with low literacy may have difficulty understanding instructions; this ultimately results in decreased adherence and poor medication management. Knowledge about diabetes mellitus and its treatments, socio-demographics, beliefs about treatment, patient-provider relationship and support received from health care services are the other factors that affect diabetic patient's adherence. 5 
The influence of non adherence to antidiabetic medications is the most important cause of uncontrolled diabetes. Several self-reported validated questionnaires were developed to monitor medication adherence in chronic disease patients including diabetes mellitus. Some of the scales suitable for measuring adherence in diabetic patients include Morisky medication adherence scale-8 (MMAS-8). ${ }^{6}$

So the study is aimed to evaluate the medication adherence among patients who are taking the antidiabetic drugs and to evaluate the additional factors effecting medication adherence like education and occupation. This study helps to determine the relationship between medication adherences to uncontrolled diabetes, compliance to medication and to improve quality of life of diabetic patients.

\section{METHODS AND MATERIALS:}

Study Design: An observational questionnaire based study was carried out for a period of six months from September 2019- February 2020.

Designing of Questionnaire: Validated MMAS-8 questionnaire was used to evaluate the medication adherence of the patients to the antidiabetic drugs. The questionnaire consists of 8 questions in which first 7 closed ended questions indicate the patient's adherence and nonadherence to the medications. The 8th question is 5 item Likert scale which determines how often the patient does not take their medications. ${ }^{6}$

Inclusion and Exclusion Criteria: a) All the diabetic patients admitted in medical departments of hospital were included in the study. b) The IP patients with diabetes mellitus from all medical departments were included in the study. c) Patients who were not willing to participate were excluded in the study.

Participant Consent: As it is mandatory to take consent from the participant for their involvement in the study as per ICH guidelines, consent was taken from the respondents using Participant Consent Form. The designed patient consent form and the MMAS-8 questionnaire were translated in to the local languages Kannada and Telugu.

Data Collection and Compilation: Project team approached 100 respondents in the NMCH. The respondents were requested to complete the MMAS-8 questionnaire and hand over immediately. Before giving the questionnaire the patients or the bystanders were signed with the participant consent form and the patients who were not willing to cooperate with the study were excluded. The questionnaire was translated to local languages like Kannada and Telugu for the easy understanding of the patient. Questions have been asked orally to some of the respondents and recorded. The filled questionnaires were evaluated as per the study objectives. The patient medication adherence determined based upon the scores obtained from the MMAS-8. According to the scores obtained medication adherence were categorized into 3 groups, that is high adherence (score 8), moderate adherence (score 6-8) and low adherence (score $<6)$. The comparison of medication adherence with various parameters such as sex, age distribution, socioeconomic data, marital status, educational qualification and occupation were noted.

Statistical Analysis: The demographic data, disease data and drug data of the patients were analyzed. Data was analyzed using descriptive statistics namely total numbers, percentage, to represent medication adherence. Microsoft word and Excel have been used to generate graphs, tables etc. All gathered data were entered into SPSS software for windows, the chi square test were applied to compare the gathered data between the two groups of MMAS-8 score and also for the comparison of data used in the study.

\section{RESULTS AND DISCUSSION}

\section{Results:}

Comparison of demographic characteristics between low and moderate/high adherence groups among 100 diabetic patients.

- Based on comparison of adherence based on the gender, majority of patients were males and males have more adherence to medications compared to females.

- Based on the age distribution it was seen that patients with age group of 41-60 years are more and have high adherence than other age groups.

- Based on marital status married patients were more than divorced and single/unmarried patients.

- Based on education of the patient, the illiterate patients were more and have lesser adherence. But the graduates were less and have more adherences to medications. There were a significant relation found between education and adherence.

- Based on the occupation of the patient, house wife were more and have lesser adherence. There were a significant relation found between occupation and adherence.

Table 1: Comparison of adherence to medication based on marital status $(n=100)$

\begin{tabular}{|c|c|c|c|c|c|}
\hline SL. NO. & GENDER & NO. OF PATIENT & LOW ADHERENCE & MODERATE/HIGH ADHERENCE & P VALUE \\
\hline 1 & Male & $\mathbf{6 2 ( 6 2 \% )}$ & $\mathbf{4 6 ( 4 6 \% )}$ & $\mathbf{1 6 ( 1 6 \% )}$ & 0.955 \\
\hline 2 & Female & $\mathbf{3 8 ( 3 8 \% )}$ & $\mathbf{2 8 ( 2 8 \% )}$ & $\mathbf{1 0 ( 1 0 \% )}$ \\
\hline
\end{tabular}

Table 2: Comparison of adherence to medication based on age $(n=100)$

\begin{tabular}{|c|c|c|c|c|c|}
\hline SL. NO & AGE & NO. OF PATIENT & LOW ADHERENCE & MODERATE/HIGH ADHERENCE & P VALUE \\
\hline 1 & $21-40$ & $18(18 \%)$ & $10(10 \%)$ & $8(8 \%)$ & 0.1323 \\
\hline 2 & $41-60$ & $58(58 \%)$ & $46(46 \%)$ & $12(12 \%)$ & \\
\hline 3 & $61-80$ & $24(24 \%)$ & $18(18 \%)$ & $6(6 \%)$ & \\
\hline
\end{tabular}


Table 3: Comparison of adherence to medication based on marital status $(n=100)$

\begin{tabular}{|c|c|c|c|c|c|}
\hline SL. NO. & MARITAL STATUS & NO. OF PATIENT & LOW ADHERENCE & MADERATE/HIGH ADHEREANCE & P VALUE \\
\hline 1 & Unmarried & $\mathbf{2 6 ( 2 6 \% )}$ & $\mathbf{1 6}(16 \%)$ & $\mathbf{1 0}(10 \%)$ & 0.0921 \\
\hline 2 & Married & $\mathbf{7 4 ( 7 4 \% )}$ & $\mathbf{5 8 ( 5 8 \% )}$ & $\mathbf{1 6}(16 \%)$ & \\
\hline
\end{tabular}

Table 4: Comparison of adherence to medication based on education $(n=100)$

\begin{tabular}{|c|c|c|c|c|c|}
\hline SL. NO. & EDUCATION & NO. OF PATIENT & LOW ADHERENCE & MODERATE/HIGH ADHERENCE & P VALUE \\
\hline 1 & None & $14(14 \%)$ & $14(14 \%)$ & $0(0 \%)$ & \multirow{2}{*}{0.0000002} \\
\cline { 1 - 5 } 2 & Primary & $26(26 \%)$ & $22(22 \%)$ & $4(4 \%)$ & $6(6 \%)$ \\
\hline 3 & Secondary & $38(38 \%)$ & $32(32 \%)$ & $16(16 \%)$ & \\
\hline 4 & Graduate \& above & $22(22 \%)$ & $6(6 \%)$ & \\
\hline
\end{tabular}

Table 5: Comparison of adherence to medication based on occupation $(n=100)$

\begin{tabular}{|c|c|c|c|c|c|}
\hline SL. NO. & OCCUPATION & NO. OF PATIENT & LOW ADHERENCE & MODERATE/HIGH ADHERENCE & P VALUE \\
\hline 1 & Farmers/Agricultures & $19(19 \%)$ & $18(18 \%)$ & $1(1 \%)$ & \\
\cline { 1 - 5 } 2 & Business & $12(12 \%)$ & $8(8 \%)$ & $4(4 \%)$ & \multirow{2}{*}{0.0268} \\
\cline { 1 - 5 } & House wife & $33(33 \%)$ & $26(26 \%)$ & $10(10 \%)$ & \\
\hline 4 & Employees & $20(20 \%)$ & $10(10 \%)$ & $4(4 \%)$ & \\
\hline 5 & Others & $16(16 \%)$ & $12(12 \%)$ & & \\
\hline
\end{tabular}

\section{DISCUSSION:}

Medication adherence is the patient compliance to the medications. There are more chances of patient's nonadherence to the patients who are taking long term therapy. This may be due to factors such as age, gender, education, occupation and marital status of the patient. Illiteracy and lack of awareness are commonly associated with the nonadherence to the medications.

An evaluation of medication adherence was carried out by using MMAS-8 questionnaire in 100 patients who have been diagnosed with diabetes mellitus. The patient's adherence to the medications was categorized in to 3 groups (poor adherence, moderate adherence and high adherence) according to the scores achieved from the MMAS-8 questionnaire. Table 1 shows the comparison of demographic characteristics between low and moderate/high adherence groups among diabetes patients. The data suggests that out of 100 patients $62(62 \%)$ were male and $38(38 \%)$ were female in which $46(46 \%)$ of male patients had low adherence and $16(16 \%)$ of male patients were having moderate/ high adherence. In $32 \%$ of female patients $28(28 \%)$ having low adherence and $10(10 \%)$ of female patients were having moderate/high adherence. This data indicates that both the male and female patients are having low adherence irrespective of their genders.

Table 2 illustrates the comparison based on the age of the patients. The patients were categorized according to their age that is patient's lies between 21-40 years, 41-60 years and 61-80 years. A total of $18(18 \%)$ patients were between age group of 21-40 years in which $10(10 \%)$ patients had low adherence and $8(8 \%)$ patients were having moderate/high adherence. Most of the patients $58(58 \%)$ were under the age group of 41-60 years from which $46(46 \%)$ and 12 (12\%) were having poor and moderate/high adherence respectively. $18(18 \%)$ and $6(6 \%)$ of patients were having poor adherence and moderate/high adherence respectively in a total of 24 (24\%) patients between 61-80 years of age. This data shows that the patients with age group of 41-60 years are prone to have diabetes than other groups, and they likely to have little more adherence than other age group patients.

The marital status of the patients was compared between low and moderate/high adherence of the patients shows in the Table 3. The patients were categorized as unmarried and married. As depicted, there was a higher percentage of married patients 74 (74\%) followed by unmarried patients $26(26 \%)$. In 74\% of married patients most of them had low adherence 58 (58\%) and 16 (16\%) had moderate/ high adherence. Among the unmarried patients 16(16\%) \& 10 $(10 \%)$ were having poor and moderate/ high adherence respectively. The data suggests that the married patients were having least adherence to the medications than the other patients which may be due to their mental stress and carelessness.

Table 4 represents the education status of the patients. A total of $14(14 \%)$ of patients were illiterate and most of the patients had low adherence (14\%). The patients having primary education were $26(26 \%)$, in which $22(22 \%)$ of them were having low adherence and only 4 (4\%) had moderate/high adherence. $38(38 \%)$ of patients were with secondary education, from which $32(32 \%)$ patients was with low adherence and $6(6 \%)$ patients had moderate/high adherence. The patients with the qualification of graduation and above were $22(22 \%)$ but they had more adherence to medications $16(16 \%)$ compared to other patients and only 6 (6\%) had low adherence to the medications. This data indicates that education is the major for medication adherence. Awareness and sticking to the medications is the most reliable factor for medication adherence. 
Occupation of the patients was described in the Table 5. In which the comparisons were done between 5 groups. Most of the patients were house wives $33(33 \%)$ and $26(26 \%)$ of them had low adherence and $7(7 \%)$ were having moderate/high adherence. 20 (20\%) of patients were categorized in the employees groups in which both low adherence and moderate/high adherence were equal $10(10 \%) .19(19 \%)$ of the patients were farmers most of them had low adherence of $18 \%$ and $1 \%$ of farmers had moderate/ high adherence.16 (16\%) of patients were categorised in other group which include government employees, merchants etc. in which most of them 12 (12\%) were having low adherence and 4 (4\%) of patients had moderate/high adherence. The rest of $12 \%$ patients were included in the business category in which $8 \%$ and $4 \%$ had low and moderate/high adherence respectively.

Statistical analysis for the evaluation of the medication adherence was done. In which Table 1 shows the comparison of the adherence between the genders. There was no significance with in the gender, which means that the adherence to the medications for drugs is lesser with irrespective of their gender. Table 2 shows the comparison of the medication adherence with their ages and there were no significance shows which indicates age is not contribute to the patient medication adherence in this study. Table 3 shows the comparison of medication adherence with the marital status of the patient, this data also shows nothing significant. Table 4 illustrates the comparison of adherence with the education of the patient. This result shows the education of the patient shows significance (0.0000002) in the medication adherence to the patient. Patient's awareness and knowledge contributes to the improved medication adherence. Table 5 shows the comparison of the patient adherence based upon their occupation which also shows significance (0.0268). The occupation of the patient contributes to the patient education adherence in this study. The same result was also reported in Kumar AA et al. ${ }^{6}$

\section{CONCLUSION:}

Diabetes mellitus is the major healthcare problem associated with high health burdens. Diabetes mellitus is the most common endocrine disorders and the leading factor for increased mortality rate. In India, the overall prevalence of diabetes mellitus is $11.8 \%$ which is rising steeply. Hence there is an increasing need of conducting studies regarding diabetes mellitus.The advent of uniquely powerful medicines and reliable means to measure adherence highlights the importance of patient adherence, particularly in diabetic patients. The adherence level to the prescribed anti-diabetic medications was found to be sub-optimal. Factors like age, gender, marital status, education, occupation are more important predictors of good adherence to anti-diabetic medications. Education is the main factor which contributes for high adherence. In our study there were a significant relation found between education and good adherence to medications. When the knowledge, and awareness increases which directly leads to good adherence to the patients. There is also a significant relation between occupation and adherence. There were no significant association found between the age, gender and marital status of the patients. Improving medication adherence in diabetic patients can helps to achieve desired goals for controlling diabetes mellitus.

\section{Acknowledgments}

Authors take it as a privilege to acknowledge Sri S R Reddy; Chairman Navodaya Educational Trust, Medical Superintendent; Navodaya Medical College Hospital and Research Center, Principal; NET Pharmacy College, HODs of department of Pharmacy practice and Medicine department and the staffs for their support during the study. Special thanks to Mr. Bhaskar, Biostatician, for his valuable guidance and suggestions throughout our project work.

\section{Conflict of Interest}

Nil.

\section{REFERENCES:}

1. Mohan V, Sandeep S, Deepa R, Shah B, Varghese C. Epidemiology of type 2 diabetes: Indian scenario. INDIAN J MED RES 2007; 125:217-30.

2. Jahagirdar SS, Bant DD, Bathija GV. Study of prevalence of diabetes mellitus in the rural areas of Hubballi,Karnataka,India. Int J Community Med Public Health 2017; 4(1):104-9.

3. Aloudah NM, Scott NW, Aljadhey HS, Araujo-Soares V, Alrubeaan KA, Watson MC. Medication adherence among patients with Type 2 diabetes: A mixed methods study. PloS one. 2018 Dec 11; 13(12):e0207583.

4. Mathew EM, Rajiah K. Assessment of medication adherence in type- 2 diabetes patients on poly pharmacy and the effect of patient counseling given to them in a multispecialty hospital. Journal of basic and clinical pharmacy. 2013 Dec; 5(1):15.

5. Aminde LN, Tindong $M$, Ngwasiri CA, Aminde JA, Njim T, Fondong AA, Takah NF. Adherence to antidiabetic medication and factors associated with non-adherence among patients with type 2 diabetes mellitus. BMC 2019; 19(35):1-9.

6. Kumar AA, Reddy V, Parveen S, Padmakar SB, Lakshmi SV, Shahzadi SY. Evaluation of Medication Adherence in Type-2 Dm Patients in a Tertiary Care Teaching Hospital.

7. Cramer JA. A systematic review of adherence with medication for diabetes. Diabetes Care 2004; 27(5):1218-24.

8. Lin LK, Sun Y, Heng BH, Chew DEK, Chong PN. Medication adherence and glycemic control among newly diagnosed diabetes patients. BMJ Open Diab Res Care 2017; 5(000429):19.24 .

9. Lee CS, Tan JHM, Sankari U, Koh YLE, Tan NC. Assessing oral medication adherence among patients with type-2 diabetes mellitus treated with polytherapy. BMJ Open 2017; 7(016317): $1-7$.

10. Dehdari L, Dehdari T. The determinants of anti-diabetic medication adherence on the experiences of patients with type 2 diabetes. Archives of Public Health. 2019 Dec 1; 77(1):21.27.

11. Grant RW, Devita NG, Singer DE, Meigs JB. Polypharmacy and medication adherence in patients with type 2 diabetes. Diabetes care. 2003 May 1; 26(5):1408-12.28.

12. Wabe NT, Angamo MT, Hussein S. Medication adherence in diabetes mellitus and self management practices among type-2 diabetics in Ethiopia. North American journal of medical sciences. 2011 Sep; 3(9):418.29.

13. Khotkar K, Chaudhari S, Jadhav P. A Deshmukh Y. Assessment of medication adherence in type II diabetic patients: A crosssectional study. MGM Journal of Medical Sciences. 2017; 4:65-9. 